\title{
Validation of a noninvasive test routinely used in otology for the diagnosis of cerebrospinal fluid shunt malfunction in patients with normal pressure hydrocephalus
}

\author{
Laurent Sakka, MD, DVM, PhD, ${ }^{1,2}$ Alexandre Chomicki, MSc, ${ }^{4}$ Jean Gabrillargues, MD, ${ }^{3}$ \\ Toufic Khalil, MD,1,2 Jean Chazal, MD, ${ }^{1,2}$ and Paul Avan, PhD, MD ${ }^{4,5}$
}

1Service de Neurochirurgie, Hôpital Gabriel Montpied, Centre Hospitalier Universitaire de Clermont-Ferrand; '2Equipe IGCNC, EA 7282, ISIT, UMR 6284, CNRS, Université d'Auvergne; ${ }^{3}$ Service de Neuroradiologie, Hôpital Gabriel Montpied, Centre Hospitalier Universitaire de Clermont-Ferrand; 'Laboratoire de Biophysique Sensorielle, Faculté de Médecine, Université d'Auvergne; and ${ }^{5}$ Biophysique Médicale, Centre Jean Perrin, Clermont-Ferrand, France

\begin{abstract}
OBJECTIVE Ventriculoperitoneal shunting is the first-line treatment for normal pressure hydrocephalus. Noninvasive auditory tests based on recorded otoacoustic emissions were assessed, as currently used for universal neonatal hearing screenings, for the diagnosis of cerebrospinal fluid shunt malfunction. The test was designed based on previous works, which demonstrated that an intracranial pressure change induces a proportional, characteristic, otoacoustic-emission phase shift.
\end{abstract}

METHODS Forty-four patients with normal pressure hydrocephalus (23 idiopathic and 21 secondary cases) were included in this prospective observational study. The male:female sex ratio was 1.44, the age range was 21-87 years (mean age 64.3 years), and the range of the follow-up period was $1-3$ years (mean 20 months). Patients were implanted with a Sophy SU8 adjustable-pressure valve as the ventriculoperitoneal shunt. The phase shifts of otoacoustic emissions in response to body tilt were measured preoperatively, immediately postoperatively, and at 3-6 months, 7-15 months, 16-24 months, and more than 24 months postoperatively. Three groups were enrolled: Group 1, 19 patients who required no valve opening-pressure adjustment; Group 2, 18 patients who required valve opening-pressure adjustments; and Group 3, 7 patients who required valve replacement.

RESULTS In Group 1, phase shift, which was positive before surgery, became steadily negative after surgery and during the follow-up. In Group 2, phase shift, which was positive before surgery, became negative immediately after surgery and increasingly negative after a decrease in the valve-opening pressure. In Group 3, phase shift was positive in 6 cases and slightly negative in 1 case before revision, but after revision phase shift became significantly negative in all cases.

CONCLUSIONS Otoacoustic emissions noninvasively reflect cerebrospinal fluid shunt function and are impacted by valve-opening pressure adjustments. Otoacoustic emissions consistently diagnosed shunt malfunction and predicted the need for surgical revision. The authors' diagnostic test, which can be repeated without risk or discomfort by an unskilled operator, may address the crucial need of detecting valve dysfunction in patients with poor clinical outcome after shunt surgery.

http://thejns.org/doi/abs/10.3171/2015.1.JNS142142

KEY WORDS normal pressure hydrocephalus; ventriculoperitoneal shunt; otoacoustic emissions; body tilt; shunt malfunction

$\mathrm{V}$ ENTRICULOPERITONEAL shunting remains the firstline treatment for normal pressure hydrocephalus $(\mathrm{NPH})$, despite a high rate of malfunctions that reaches $31 \%$ within the 2 years following surgery. ${ }^{9,12}$ Its detection is challenging since neuroimaging does not systematically correlate with clinical outcome. Notably, CT scanning often discloses the persistent distension of the cerebral ventricles in clinically improved patients. ${ }^{13,14}$ In addition, the cerebrospinal fluid (CSF) dynamic tests recommended by specialized neurosurgical teams ${ }^{6,17,21}$ are invasive and are not suitable for patients who are attended either by their general practitioner or at the emergency 
department of an institution without a neurosurgical department. Therefore, the diagnosis of shunt malfunction remains difficult.

The goal of our work was to evaluate a simple, noninvasive auditory test that assesses shunt functionality in patients with insufficient clinical results. Previous work has shown that CSF pressure changes are transmitted to the inner-ear fluids, thus influencing the mechanical loads on the cochlear basilar membrane and the stapes footplate at the boundary between the inner and middle ears. These mechanical loads can be noninvasively probed using otoacoustic emissions (OAEs), which are the sounds naturally emitted by the cochlea in response to sound stimuli. Detected in the external auditory meatus, OAEs are sensitive to changes in sound propagation through the inner and middle ears. Low-frequency OAEs have been shown to react to intracranial pressure (ICP) changes by a phase shift that is proportional to the ICP changes produced by the infusion tests ${ }^{4}$ or body tilt. ${ }^{5}$

Detecting OAEs is an audiological test that has served as a universal hearing screening of newborns for more than 20 years in many countries. ${ }^{1,10,11,22}$ Because it is fully automatized, the test is administered by unskilled personnel in maternity wards. Published studies have demonstrated that OAEs detect relative changes in ICP and can be used to monitor ICP after initial calibration. One purpose of ventriculoperitoneal shunting is to buffer ICP changes. In the case of shunt malfunction, buffering should no longer happen. Thus, we hypothesized that OAE modifications induced by a procedure that triggers slight changes in ICP could reflect the functionality of a ventriculoperitoneal shunt. We reported the results of one such protocol, whereby tilting the body from the upright to the supine posture produced moderate and reversible modulations of ICP. ${ }^{5}$ In normal subjects with stable ICP, the OAE phases are stable over months and the posture-induced OAE phase shift is positive. The biomechanical interpretation is that the increased ICP in the supine position in comparison with the upright posture increases the stiffness of the ossicular chain in relation to increased inner-ear pressure. The mechanics of the inner ear prescribes that the greater the stiffness, the more positive the OAE phase. ${ }^{4}$ The OAEs were repeatedly recorded in upright and supine postures in a series of 44 adult patients with NPH who were treated with a ventriculoperitoneal shunt over a period of 1-3 years following surgery, during which more than 200 body-tilt tests were performed with an automatically computed OAE phase shift as the readout. We examined whether this readout in shunt-treated patients differed between normal and preoperative values and was sensitive to shunt functionality and setting.

\section{Methods}

\section{Patients}

Forty-four consecutive, eligible, adult patients with NPH were included (26 males and 18 females; mean age 64.3 years; median age 68 years; range $41-87$ years). Twentythree patients had idiopathic and 21 had secondary NPH (15 cases developed after spontaneous subarachnoid hemorrhage and 6 after head trauma). Diagnosis was made on the basis of: 1) clinical symptoms, including ataxia (gait unsteadiness, $180^{\circ}$ turn in more than 3 steps), urinary incontinence, and cognitive impairment (psychomotor slowing, behavioral changes, memory disorders); 2) cerebral imaging (CT scan and MRI); and 3) the results of CSF dynamic tests (basal ICP value, resistance to CSF outflow, and pressure-volume index). A ventriculoperitoneal shunt was inserted using the Sophy SU8 adjustable-pressure valve set to $110 \mathrm{~mm} \mathrm{H}_{2} \mathrm{O}$. This valve is not fitted with an antisiphon device. After surgery, patients were followed up over a period of 1-3 years (average 20 months). During follow-up, the patients were regularly checked clinically by their neurosurgeons. When a relapse of NPH symptoms was observed after a period of improvement, the first step was to perform a CT scan to check ventriculomegaly and obtain x-rays to verify the setting of the valve and its proper connection. The second step consisted of lowering the opening pressure and following the clinical evolution. When valve disconnection was detected, a surgical procedure was decided. This decision was not influenced by the results of the OAE test, which was performed by a separate operator who did not communicate the readout to the neurosurgeon.

Group 1 included 19 patients who did not require adjustment of the valve-opening pressure throughout the whole duration of the study. For the data analysis, postural tests were pooled into successive time intervals: immediately preoperation; immediately postoperation; 3-6 months postoperation; 7-15 months postoperation; 16-24 months postoperation; and more than 24 months postoperation. All patients were tested preoperatively and a few days following surgery. The number of tests available for analysis at each later postoperative time interval varied between 10 and 11, as patients sometimes deferred a visit. The average duration over which postural tests were available was 24 months (SD 10 months; maximum duration 37 months).

Group 2 included 18 patients who required valve-opening pressure adjustment. A postural test of the OAEs was performed just before and after adjustment. The postural shifts of OAEs were tested preoperatively and a few days following surgery. The number of postural tests performed during the 3- to 6-month period was 15 , which decreased to 10 during the later intervals. The average duration over which the postural tests had been performed was 17 months (SD 9 months; maximum duration 31 months).

Group 3 included 7 patients who required valve replacement at some stage of this study. In 5 patients, surgery confirmed a shunt malfunction. In 2 patients with subdural hematoma, the shunt had been blocked deliberately before inclusion, and the aim of surgery was to replace it with a Sophy SM8-300 that was set to a higher opening pressure of $150 \mathrm{~mm} \mathrm{H}_{2} \mathrm{O}$. Thus, in all patients, the posture test done just before valve replacement did correspond to a nonfunctioning shunt. Later, the postural tests followed the postoperative schedules of Groups 1 and 2.

\section{Objective Test}

In response to sound stimulation, OAEs travel backward by the middle-ear ossicular chain from their intracochlear sources toward the microphone of the detecting 
acoustic probe placed in the external auditory meatus (Fig. 1A). ${ }^{1}$ Previous studies have reported that the amplitudes and phases of OAEs exhibit a high long-term stability in steady physiological situations, apart from a slight circadian modulation. ${ }^{3}$ The OAEs recorded in a stable situation in a subject can thus serve as a stable reference. When ICP increases with respect to this reference, the OAE phase around $1 \mathrm{kHz}$ exhibits a positive shift that is proportional to the ICP increase. ${ }^{4}$ Biophysical models show that this shift is specific to changes in the stiffness of the stapes footplate at the interface between the inner and middle ears. In the context of a concomitant ICP change, the stapes footplate likely gets pushed by the hydrostatic pressure of the cochlear fluids, thereby straining its annular ligament as the CSF and cochlear fluids equalize their pressures through the cochlear aqueduct (Fig. 1B and C). ${ }^{19}$ When OAEs travel through the stapes to the recording microphone in the external auditory meatus, their phase shifts reflect these biomechanical changes accordingly (Fig. 1D).

Here, the confounding effects of middle-ear mechanics were excluded before postural tests using tympanometric measurements to show that the air pressures were equal on both sides of the tympanic membrane. Then, OAEs were recorded with commercially available equipment (ILO88; Otodynamics), according to the routine procedure used in otology and pediatrics, in the 2 ears of the patients at 2 different ICPs provided by 2 postures (sitting upright vs supine on a horizontal bed). The operator inserted the tip of the OAE microphone into the external auditory meatus of the chosen ear, then the whole recording procedure proceeded automatically. The first OAE recording was performed after the patient had been supine for at least 5 minutes, first in the left ear and then the right ear. Each recording took between 45 and 75 seconds. Next, the patient was moved to an upright posture by way of the bed's mechanism. Two minutes later, the OAEs were collected in the right ear, and then in the left ear 2 minutes later.

In healthy subjects and adequately shunt-treated patients, the CSF pressure changes induced by body tilt stabilize in a few seconds. Then, pressure equalization through the cochlear aqueduct occurs within a time constant of a few tens of seconds..$^{19}$ Thus, there was ample time for the pressures of the CSF and cochlear fluid to equalize and fully reflect the effects of body tilt before the right ear was measured after body tilt, and the right and left OAE shifts should be identical. This might not hold true in patients with malfunctioning shunts where ICP buffering is likely delayed or insufficient. Here, it was expected that delayed ICP buffering would translate to right-left asymmetry in OAE shifts, and the OAE recordings were performed in the right ear before the completion of pressure buffering. The 2 ears were thus processed separately.

Postural tests were scheduled the day before surgery, 1-5 days after surgery, 3-6 months later, and then approximately every $6-12$ months. The confounding effects of age-related impairment on cochlear physiology were avoided by taking into account only the postural phase shift that occurred within a few minutes in an ear that served as its own control. The only age-related prerequisite for phase shift to be measured was the stable presence
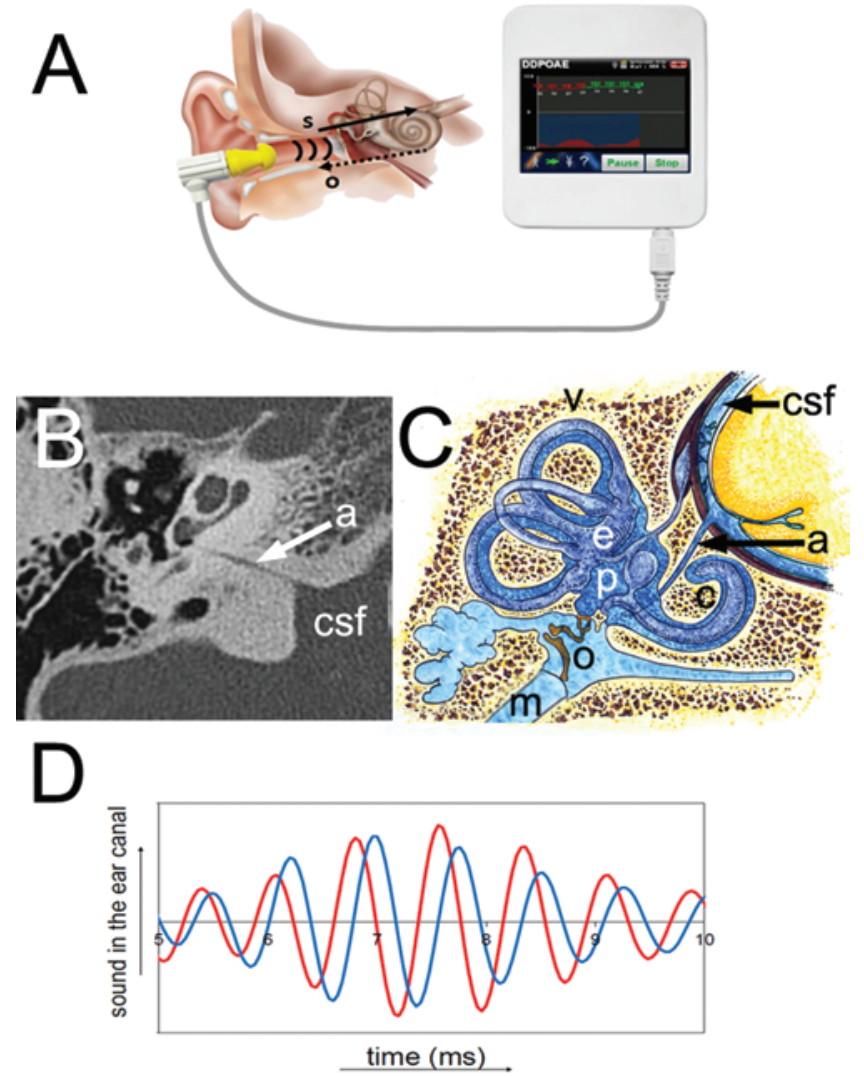

FIG. 1. A: Principle of OAE recording. The tip of the probe containing an earphone and a microphone (left) is tightly sealed in the external auditory meatus. A sound stimulus (s; solid arrow) is emitted, and the OAE (o; dashed arrow) is reemitted backward by the cochlear sensory cells. The OAE recording device (right) controls the sound stimulus and detects the OAE (around $1 \mathrm{kHz}$ for ICP-monitoring purposes). Its phase is automatically extracted and plotted on the screen. B: CT scan of the right temporal bone. The cochlear aqueduct (a) connects the CSF of the subarachnoid space to the perilymphatic fluid of the cochlea. C: Anatomical sketch of the middle ear, labyrinthine, and CSF spaces (shown in different shades of blue; $e=$ endolymph; $p=$ perilymph) and their relationships $(a=$ cochlear aqueduct; $c=$ cochlea; $m=$ external auditory meatus; $0=$ middle-ear ossicles; $v$ = vestibule). Copyright Paul Avan. Published with permission. D: Plot of the sound pressure in the external auditory meatus showing an example of raw $\mathrm{OAE}$ around $1 \mathrm{kHz}$ in the upright posture (blue) and supine posture (red). Although the 2 plots have similar amplitudes and envelopes, the red plot is shifted to the left by about $0.25 \mathrm{~ms}$, which reveals a phase shift of $+90^{\circ}$. Figure is available in color online only.

of OAEs around $1 \mathrm{kHz}$. This required either normal hearing thresholds or sensorineural hearing loss not exceeding $30 \mathrm{~dB}$ around $1 \mathrm{kHz}$. As presbycusis predominantly affects frequencies well above $1 \mathrm{kHz}$, many ageing patients have useful OAEs at $1 \mathrm{kHz}$. With the help of homemade software, the phase shift was automatically computed for the spectral OAE component to the nearest $1 \mathrm{kHz},{ }^{5}$ with the upright posture arbitrarily serving as reference. In the time domain, a positive phase shift corresponded to a time lag of the OAE waveform in the upright posture relative to that in the supine posture.

This noninterventional, observational study complied with ethical rules (EudraCT number registration, AFSSAPS French agency: 2010-A00266-33; approval by the 
local ethics committee: CPP Sud-Est VI AU 839), and the informed consent of the patients was obtained before inclusion. The recruitment period lasted 4 years from approval and included all consenting patients who had detectable OAEs around $1 \mathrm{kHz}$ (i.e., 44 of 65 consenting NPH patients).

\section{Statistical Analysis}

The series of measurements of postural phase shift of OAEs at $1 \mathrm{kHz}$ in degrees were compared after testing for the normality and homoscedasticity of data distributions. Two-way ANOVA ("time" and "side") was used in the first group of patients in whom multiple comparisons had to be performed among 6 different measurement times, in the right and left ears. Post hoc comparisons were done using the Bonferroni t-test, which presents the least risk of wrongly detecting spurious differences. In Group 2 , only 3 measurement times were considered: preoperative and before and after valve-pressure adjustments. Bilateral paired Student t-tests were deemed more suitable for performing the comparisons, with the Bonferroni correction that adjusts the $\mathrm{p}$ values as a function of the number of comparisons (i.e., 3) in order to keep the Type I errors under control. The right and left ears were tested independently, as it was expected that the time at which phase shift was measured, suitable in the left ear, might have been premature in the right ear in case of delayed ICP buffering. For the group of 7 patients who underwent surgical shunt valve replacement because the previous valve was blocked (i.e., Group 3), the pre- versus postoperative comparison was made with the help of the unilateral test as the direction of postural changes, if any, was predictable. The threshold for the significance of the p value was set at 0.05 .

\section{Results \\ Group 1}

In Group $1(n=19)$, the initial valve-opening pressure set to $110 \mathrm{~mm} \mathrm{H}_{2} \mathrm{O}$ was unchanged over the more than 2-year-long follow-up. The average phase shift in the left ear changed from $+11.1^{\circ}$ (SEM $3.4^{\circ}$ ) before surgery to $-11.6^{\circ}\left(\mathrm{SEM} 6.1^{\circ}\right.$ ) just after surgery. In the right ear (tested earlier after body tilt), similar phase shifts were observed, changing from $+9.1^{\circ}$ (SEM $7.7^{\circ}$ ) before surgery to $-7.1^{\circ}$ (SEM 5.5 ) just after surgery (Fig. 2). Two-way ANOVA disclosed the significant effect of time $(p=0.007)$, no difference between the right and left ears $(\mathrm{p}=0.435)$, and no interaction between time and side $(\mathrm{p}=0.984)$. Post hoc comparisons showed significant differences only between preoperative phase shift, on one hand, and all postoperative phase shifts, on the other hand, regardless of side. Thus, the postoperative phase shifts were stable in both ears without being significantly influenced by postoperative delay (Fig. 2). For example, in the left ear, the average phase shift after more than 24 months was still $-14.2^{\circ}$ (SEM $6.8^{\circ}$ ). In summary, persistent inversion of the sign of the phase shift is the main effect of surgery in this group.

\section{Group 2}

In Group $2(\mathrm{n}=18)$, clinical deterioration in regards to gait disturbance, urinary incontinence, or mental decline

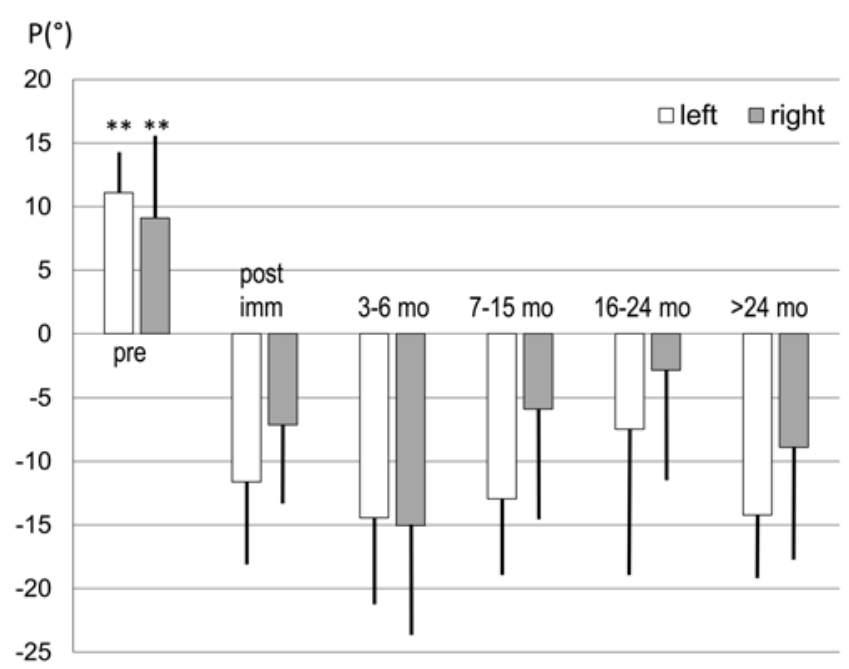

FIG. 2. Histograms of average postural phase shifts in degrees $\left({ }^{\circ}\right)$ and SEMs (vertical lines) of the 19 patients in Group 1: preoperatively (pre; $n$ $=19$ ), immediately after shunt placement (post imm; $n=19$ ), and at later time intervals ( $3-6$ months: $n=11 ; 7-15$ months: $n=11 ; 16-24$ months: $n=11 ;>24$ months: $n=10$ ) (left ear indicated by open bars; right ear indicated by shaded bars). Only the preoperative averages were significantly different from all other averages $\left({ }^{* *} 0.001<p<0.01\right)$.

required valve-opening pressure adjustments, which decreased in all cases. The average phase shift before surgery was positive $-+14.2^{\circ}$ in the left ear (SEM 5.4) and $+21.3^{\circ}$ in the right ear (SEM 9.2) - but not significantly different from the phase shift in normal subjects (which was previously reported $d^{2}$ to be $+9.7^{\circ}$ ) or patients in Group 1. The valve was initially set at $110 \mathrm{~mm} \mathrm{H}_{2} \mathrm{O}$, as in Group 1. At the first postoperative postural test, the average phase shift in the left ear changed signs to $-26.5^{\circ}$ (SEM 7.4; $\mathrm{p}=$ 0.001 and significantly different relative to the preoperative value; paired t-test); but, in the right ear, phase shift remained positive although it decreased relative to the preoperative value to $1.9^{\circ}$ (SEM 8.5; $p=0.129$ and nonsignificant difference relative to the preoperative value; paired t-test) (Fig. 3).

The valve-opening pressure changed between the 3rd and 6th months in 7 patients, and between the 7 th and the 24th months in 11 patients. The valve-opening pressure decreased in all cases - to 95 or $80 \mathrm{~mm} \mathrm{H}_{2} \mathrm{O}$-to increase CSF flow through the shunt. As a result, the average phase shift in the left ear became $-37.0^{\circ}$ (SEM 6.5) and $-7.2^{\circ}$ (SEM 10.2) in the right ear (Fig. 3). On the left side, the change in the phase shift induced by the decrease in valveopening pressure was significant $(\mathrm{p}=0.02$; paired $\mathrm{t}$-test), whereas, on the right side, the same observed trend was not significant $(p=0.20)$. Based on the apparent stability of the phase shifts across time in the first group of patients whose valve setting was unchanged, the outcomes of all tests at each opening pressure were pooled, and the grandaveraged phase shift was computed. When the valve-opening pressure was $110 \mathrm{~mm} \mathrm{H}_{2} \mathrm{O}$, the phase shift was $-16.6^{\circ}$ (SEM 6.7) in the left ear and $+4.8^{\circ}$ (SEM 6.0) in the right ear, which decreased to $-30.0^{\circ}$ (SEM 7.4) in the left ear and $-12.5^{\circ}$ (SEM 10.0) in the right ear at lower opening pressures. Again, a statistically significant difference was found between the grand-averaged phase shifts at the 2 
$\mathrm{P}\left({ }^{\circ}\right)$

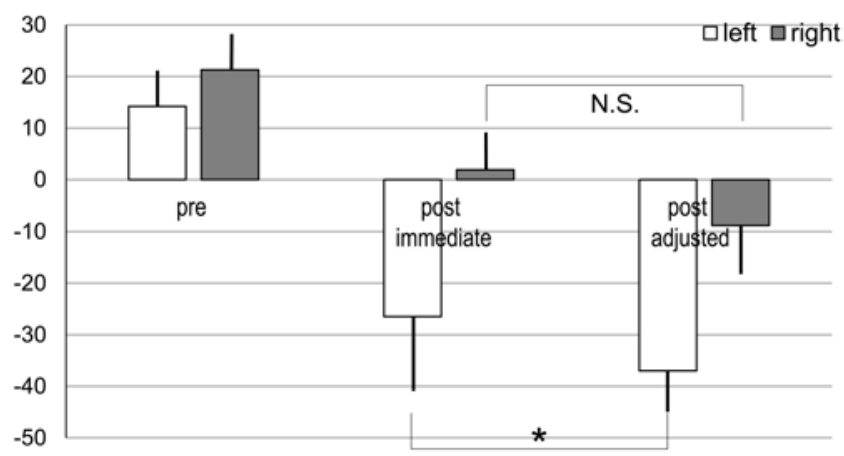

FIG. 3. Histograms of average postural phase shifts in degrees $\left({ }^{\circ}\right)$ and SEMs (vertical lines) of the 18 patients in Group 2: preoperatively (pre), soon after shunt placement (post immediate), and after the opening pressure of the valve had been decreased (post adjusted) (left ear indicated by open bars; right ear indicated by shaded bars). The adjustment of the valve-opening pressure significantly modified the average phase shift only in the left ear $\left({ }^{*} 0.01<p<0.05\right)$, but not the right ear $(p>0.05)$. N.S. $=$ nonsignificant.

valve-opening pressures on the left side $(\mathrm{p}=0.015$; paired $\mathrm{t}$-test), but not on the right side $(\mathrm{p}=0.168)$. These results unveil striking interaural differences between phase shifts at both opening pressure settings, with smaller effects of posture in the right ear, which was always measured 2-3 minutes earlier than the left ear just after body tilting $(\mathrm{p}=$ 0.024 at $110 \mathrm{~mm} \mathrm{H}_{2} \mathrm{O}$ opening pressure; $\mathrm{p}=0.034$ at lower opening pressure; paired t-tests) (Fig. 4). Yet, no significant left-right asymmetry was present preoperatively, and in the first group of patients the phase shift measurements remained symmetrical after shunt surgery.

\section{Group 3}

Among the patients in Group $3(n=7)$ whose valves were replaced, phase shift just before revision was positive in 6 cases and slightly negative in 1 case. A few days after revision, phase shift became negative in all cases and more negative in the case that was slightly negative preoperatively. The average phase shift of the 7 reoperated patients was $+12.3^{\circ}$ (SD 20.6) in the left ear just before revision, and decreased to $-41.3^{\circ}$ (SD 24.9) after revision ( $p$ $<0.05$ ). In the right ear, phase shift decreased from $+5.8^{\circ}$ (SD 17.8) before revision to $-25.5^{\circ}$ (SD 21.5) after revision $(\mathrm{p}<0.02)$.

\section{Illustrative Cases}

One example of the immediate and delayed consequences on the phase shift of an opening-pressure adjustment is depicted in Fig. 5. In this patient in Group 2, the initial opening pressure of $110 \mathrm{~mm} \mathrm{H}_{2} \mathrm{O}$ resulted in little immediate postoperative change in phase shift. After 4 months, phase shift became even slightly positive, and the clinical signs led to a decrease in the valve-opening pressure to $80 \mathrm{~mm} \mathrm{H}_{2} \mathrm{O}$. As an immediate result, phase shift decreased to a clearly negative value and remained approximately stable in the 3 controls for the next 18 months.

Figure 6 depicts a typical example, wherein phase shift was near 0 before the initial CSF shunt placement that was

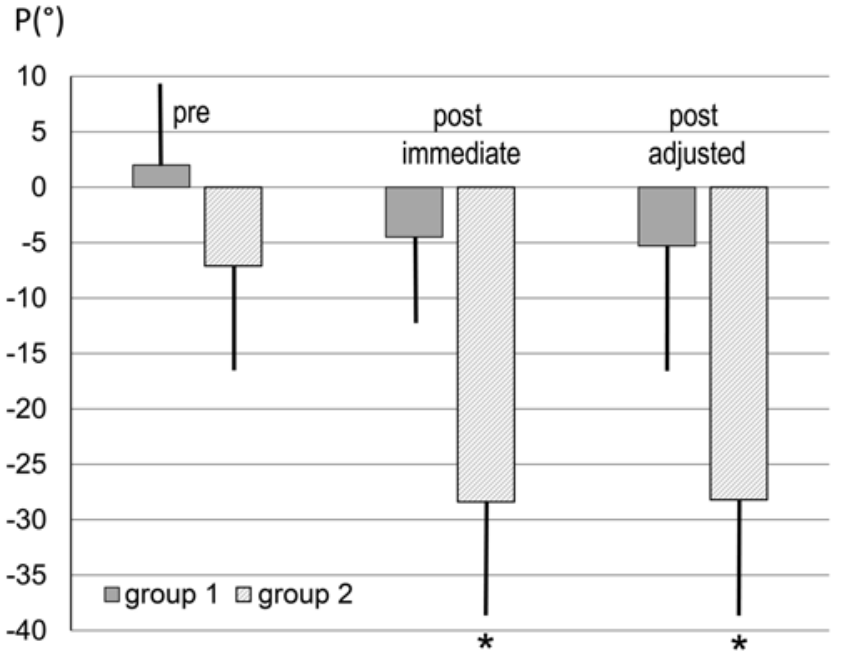

FIG. 4. Left-right asymmetry of the postural phase shift between preoperatively (pre) and soon after shunt placement (post immediate) with the valve-opening pressure set to $110 \mathrm{~mm} \mathrm{H}_{2} \mathrm{O}$ in both groups of patients, and after its decrease in Group 2 patients (post adjusted). The latter asymmetry is compared with that measured in the Group 1 patients more than 18 months after surgery. The left-right asymmetry is significant only in Group 2 patients after shunt placement $\left({ }^{*} 0.01<p<0.05\right)$.

performed at time $\mathrm{t}=0$, then became clearly negative just after surgery, and was positive 3 months later. The unsatisfactory clinical outcome suggested shunt malfunction, and so did its falling within the range of the phase shifts of nonshunted subjects. Surgical revision confirmed shunt obstruction. Just after the placement of a new valve set to $130 \mathrm{~mm} \mathrm{H}_{2} \mathrm{O}$, phase shift underwent a small decrease that was still present 2 months later. The opening pressure was then adjusted to $110 \mathrm{~mm} \mathrm{H}_{2} \mathrm{O}$, which resulted in a clearly negative and stable phase shift.

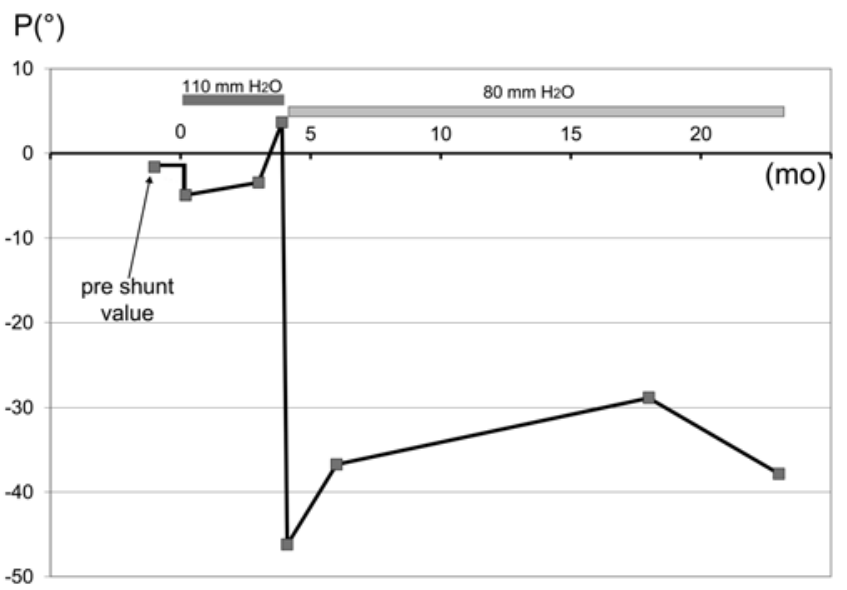

FIG. 5. Time course of the postural phase shift in the left ear of a patient in Group 2. With the first valve pressure set to $\left(110 \mathrm{~mm} \mathrm{H}_{2} \mathrm{O}\right)$, $\mathrm{P}$, which was initially slightly lower than before surgery, became positive after 4 months. The pressure setting was decreased to $80 \mathrm{~mm} \mathrm{H}_{2} \mathrm{O}$ in an attempt to increase CSF flow through the shunt. As a result, phase shift became significantly negative and remained stable over the next 18 months. 


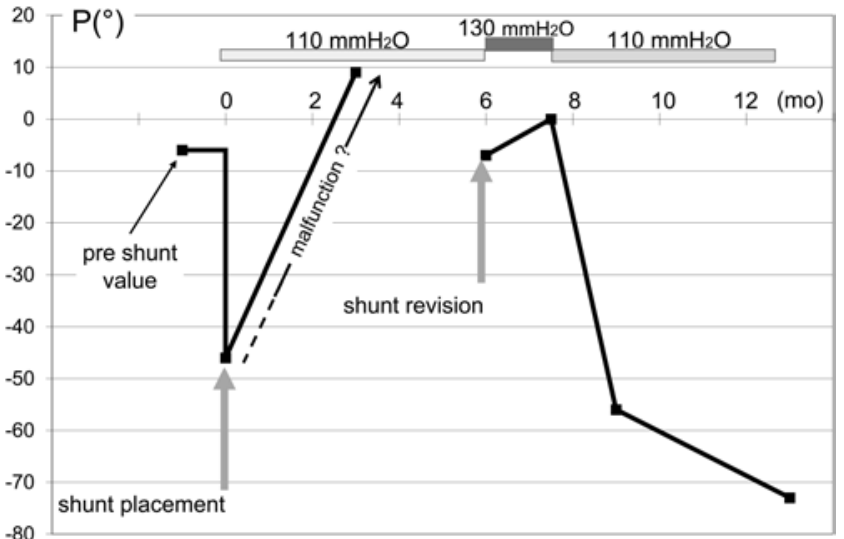

FIG. 6. Time course of the postural phase shift in the left ear of a patient who underwent surgical revision. The first shunt was placed at time $t=$ 0 . At time $t=6$ months, the clinical signs of malfunction led to shunt revision, which confirmed the obstruction of the valve. When the shunt was properly functioning, phase shift was correlated to the valve-opening pressure (in $\mathrm{mm} \mathrm{H}_{2} \mathrm{O}$ ).

\section{Discussion}

The salient findings of the present work are that the presence of a shunt, which in the immediate postoperative period is expected to be functioning, results in a negative OAE postural phase shift, in contrast with the positive preoperative phase shift observed in non-shunt-treated patients. In the absence of any sign of shunt malfunction, phase shift remained stable and negative over more than 2 years. Decreasing the opening pressure of a shunt resulted in a decrease of phase shift to a more negative plateau. Conversely, phase shift had come back to the preoperative reference in cases with proven shunt breakdown. All 44 patients had received the same model of shunt (except in 2 valve replacements), in which the valve opens when the pressure difference between inlet and outlet exceeds a preset limit. The valve-opening pressure can be noninvasively adjusted according to the clinical or anatomical status of the patient. The observed postural patterns capitalized on the repetition over several years when 75 bilateral OAE tests were performed in patients in whom a stable valve-opening pressure was maintained, whereas in 18 patients in whom a decrease in opening pressure was applied, bilateral controls were performed 3 times, i.e., just before, just after, and several months after the decrease. The postural patterns suggest a promising reliable method for testing shunt function. Even in the absence of preoperative data, the mere finding of positive phase shift in a shunt-treated patient may contribute to the diagnosis of shunt malfunction.

The present application of OAEs for the assessment of CSF flow through a ventriculoperitoneal shunt rests on the presence of the cochlear aqueduct, which is a narrow channel through the petrous part of the temporal bone that connects the subarachnoid space of the posterior cranial fossa with the perilymphatic space of the cochlea (Fig. 1B and C). Its patency, which has been reported in $93 \%$ of adult patients, ${ }^{8}$ has been invoked to explain the fine modulation of audiological test outcomes by ICP.4,16 In former studies reporting the interest of OAEs in ICP monitoring,
OAEs were recorded using invasive hydrodynamic tests during which ICP was known. ${ }^{4}$ In the present work, body tilt was used to produce a likely but small and not precisely controlled ICP change, though this is repeatable at will without any risk or discomfort. The fact that patients were their own controls circumvented the lack of precise knowledge of the applied ICP change.

One practical advantage of the proposed method stems from it being derived from a routine audiological test for which simple automatic equipment is widespread, as neonatal hearing screening based on OAEs has been implemented for years in many countries. ${ }^{22}$ Adjusting the OAE test for the goal of tracking ICP changes is allowed by simple software extensions that automatically extract the phases of the OAE components detected around $1 \mathrm{kHz}$ (Fig. 1A and D). Performing an OAE test only requires the placement of an earplug in the ear canal, as usually done in maternity wards by students or pediatric nurses with no particular knowledge of otology and after minimal technical training.

A second interest of the OAE method rests on its ability to provide physiologically meaningful information regarding ICP dynamics. In the first group of patients with a stable valve-opening pressure setting, postural phase shift displayed stable changes relative to their preoperative values. CSF diversion from the intracranial compartment to another compartment is expected to affect both the intracranial and intracochlear pressures, as the cochlear aqueduct allows these 2 pressures to equalize and thus the ear's mechanics react to the presence of the shunt. Normally, the ossicular chain is stiffer in the supine posture (i.e., positive phase shift; Fig. 1D) because an increased ICP, by pushing the stapes outwards, stretches and stiffens its annular ligament. In shunt-treated patients, negative phase shift indicates that the ossicular chain is stiffer in the upright than the supine posture. CSF flow is expected through the shunt in the upright posture as the valve opens through the effect of gravity, and this might decrease intracochlear pressure enough to displace the stapes inward, thereby stretching and stiffening its annular ligament. The concept of decreased intracochlear pressure was invoked previously to account for cochlear impairment, sensorineural deafness after shunt surgery, and attendant changes in the mechanics of the cochlea. ${ }^{20}$

In Group 2, adjusting the valve-opening pressure revealed that the smaller the valve-opening pressure, the larger the change in phase shift relative to the preoperative status. The sensitivity of phase shift to the valve settings suggests a functional shunt. Conversely, shunt malfunction, as in the group of 7 patients whose defective valves had to be replaced (i.e., Group 3), led to positive phase shift before revision surgery in 6 cases before shunt placement, thus suggesting that the shunt did not function. After revision surgery, phase shift went back to a clearly negative value in all cases, as could be expected.

During the follow-up of NPH patients, the identification of shunt malfunction is difficult because in NPH valve dysfunction does not result in the development of intracranial hyperpressure. The diagnosis is usually suspected after a phase of clinical improvement by the recurrence of symptoms of NPH or ventriculomegaly, despite valve-opening 
pressure adjustments. While normal ventricular size is not enough to rule out shunt obstruction in the presence of clinical features consistent with $\mathrm{NPH},{ }^{23}$ alternatively the shunt might work correctly despite ventriculomegaly ${ }^{13,14}$ or poor clinical outcome, since a neurodegenerative disease might progress in parallel. It follows that shunt functionality calls for complementary explorations beyond clinical and CT scan data. In this context, CSF dynamic tests performed on specialized structures provide strong arguments for the diagnosis of shunt functionality., ${ }^{6,17,21}$ CSF dynamic tests are facilitated by valves fitted with a prechamber or when an Ommaya reservoir has been inserted before shunt placement. The results of infusion tests in shunt-treated patients have been extensively detailed in situations with either a functioning ${ }^{13,15,21}$ and nonfunctioning shunt, ${ }^{6,18}$ but they remain invasive procedures performed only in a department of neurosurgery where they need to be confronted with presurgical tests. ${ }^{21}$ Therefore, a simple noninvasive test to assess CSF shunt functionality appears necessary for patients who are attended by their general practitioner, neurologist, or at an emergency department at an institution without a neurosurgical department and have no access to invasive diagnostic procedures. This requirement is satisfied by the effect of body tilt on OAEs, which have been routinely tested noninvasively, and, in the present work, efficiently detected whether a shunt and its pressure-adjusting device were functional or blocked.

A broader purpose would be to use OAEs to predict the clinical benefit or define the best setting for the valve. The lack of differences in the phase shifts between Groups 1 and 2, despite their different clinical outcomes (smooth for Group 1, eventful for Group 2), suggests that the size of the phase shift has little or no predictive value in this respect. Another outcome-the significant interaural asymmetry of the effect of body tilt regardless of pressure setting in Group 2 (Fig. 3) - may open a more promising avenue of research in this direction. We assume that after body tilt, the phase shift of the OAEs-before it reaches a plateau-follows an exponential time course defined by $\mathrm{k} \exp (-\mathrm{t} / \tau)$, as previously proposed. ${ }^{7}$ It is usually thought that the plateau of an exponential is almost reached after 5 times its half-life (i.e., $0.693 \tau$ ). The time constant $\tau$ (a few tens of seconds ${ }^{7}$ ) depends on brain compliance and, in shunt-treated patients, on the hydraulic resistance of the shunt and peritoneal-cavity system. Because the right ears were tested 2 minutes earlier than the left ears, phase shift was measured farther from the plateau. This explains why some degree of interaural difference was noted in Groups 1 and 3 after revision surgery, though this difference was small and not significant because $\tau$ is short. In Group 2, the significant interaural difference in phase shift suggests that $\tau$ increased, so the OAE measurement occurred well before the plateau in the right ear. When the opening pressure of the valve is decreased, the time course of the phase shift of OAEs is now described by $k^{\prime} \exp (-t / \tau)$, where $\mathrm{k}^{\prime}>\mathrm{k}$, so phase shift becomes more negative than under the previous pressure setting. Nevertheless, with an unchanged $\tau$ value, the same problem persists of a premature measurement in the right ear. It may, thus, be important to consider that the magnitude and time course of the flow of CSF through the shunt are 2 independent factors that influence the shunt outcome concomitantly, and pay more attention to a possibly abnormally slow time course in Group 2. Here, the appropriate measurement time corresponded to the left ear. In a generalized version of the test, any ear could be tested (e.g., the one with the largest OAEs), but with the important caveat that at least 4 to 5 minutes must have elapsed after body tilt before OAEs can be measured again.

\section{Conclusions}

Our noninvasive test based on OAE recordings reflects the functioning of the ventriculoperitoneal shunt. A longlasting inversion of OAE postural phase shift seems to be characteristic of the proper functioning of the shunt, and adjusting the valve-opening pressure impacts the postural phase shift. In our cohort of patients, OAEs consistently diagnosed shunt malfunction and confirmed the need for surgical revision; the aim of the OAE tests is not to predict the clinical efficiency of shunt placement. NPH predominantly affects elderly patients in whom the causes of comorbidity might sufficiently explain unsatisfactory outcomes after shunt placement. Thus, the management of these patients by their practitioner would benefit from a simple test to screen for valve dysfunctions. Our diagnostic test, which can be repeated by unskilled personnel without risk or discomfort, may address this crucial need before referring the patient to a neurosurgery department where invasive tests can be performed.

\section{Acknowledgment}

The technical developments for this study were partly funded by a grant (08-ETEC-001-01) from the Agence Nationale de la Recherche.

\section{References}

1. Avan P, Büki B, Petit C: Auditory distortions: origins and functions. Physiol Rev 93:1563-1619, 2013

2. Avan P, Giraudet F, Chauveau B, Gilain L, Mom T: Unstable distortion-product otoacoustic emission phase in Menière's disease. Hear Res 277:88-95, 2011

3. Bell A: Circadian and menstrual rhythms in frequency variations of spontaneous otoacoustic emissions from human ears. Hear Res 58:91-100, 1992

4. Büki B, Avan P, Lemaire JJ, Dordain M, Chazal J, Ribári O: Otoacoustic emissions: a new tool for monitoring intracranial pressure changes through stapes displacements. Hear Res 94:125-139, 1996

5. Büki B, Chomicki A, Dordain M, Lemaire JJ, Wit HP, Chazal J, et al: Middle-ear influence on otoacoustic emissions. II: contributions of posture and intracranial pressure. Hear Res 140:202-211, 2000

6. Czosnyka ZH, Czosnyka M, Pickard JD: Shunt testing in-vivo: a method based on the data from the UK shunt evaluation laboratory. Acta Neurochir Suppl 81:27-30, 2002

7. de Kleine E, Wit HP, van Dijk P, Avan P: The behavior of spontaneous otoacoustic emissions during and after postural changes. J Acoust Soc Am 107:3308-3316, 2000

8. Gopen Q, Rosowski JJ, Merchant SN: Anatomy of the normal human cochlear aqueduct with functional implications. Hear Res 107:9-22, 1997

9. Hebb AO, Cusimano MD: Idiopathic normal pressure hydrocephalus: a systematic review of diagnosis and outcome. Neurosurgery 49:1166-1186, 2001 
10. Kemp DT: Stimulated acoustic emissions from within the human auditory system. J Acoust Soc Am 64:1386-1391, 1978

11. Kemp DT, Bray P, Alexander L, Brown AM: Acoustic emission cochleography-practical aspects. Scand Audiol Suppl 25:71-95, 1986

12. Larsson A, Wikkelsö C, Bilting M, Stephensen H: Clinical parameters in 74 consecutive patients shunt operated for normal pressure hydrocephalus. Acta Neurol Scand 84:475482, 1991

13. Maksymowicz W, Czosnyka M, Koszewski W, Szymanska A, Traczewski W: The role of cerebrospinal compensatory parameters in the estimation of functioning of implanted shunt system in patients with communicating hydrocephalus (preliminary report). Acta Neurochir (Wien) 101:112-116, 1989

14. Meier U, Paris S, Gräwe A, Stockheim D, Hajdukova A, Mutze S: Is there a correlation between operative results and change in ventricular volume after shunt placement? A study of 60 cases of idiopathic normal-pressure hydrocephalus. Neuroradiology 45:377-380, 2003

15. Petrella G, Czosnyka M, Keong N, Pickard JD, Czosnyka Z: How does CSF dynamics change after shunting? Acta Neurol Scand 118: 182-188, 2008

16. Phillips AJ, Farrell G: The effect of posture on three objective audiological measures. Br J Audiol 26:339-345, 1992

17. Schuhmann MU, Schneekloth CG, Klinge P, Engel M, Samii $\mathrm{M}$, Brinker T: Dynamic shunt testing applying short lasting pressure waves-inertia of shunt systems. Acta Neurochir Suppl 81:19-21, 2002

18. Taylor R, Czosnyka Z, Czosnyka M, Pickard JD: Factors determining mean ICP in hydrocephalic patients with Hakim-programmable valve: implications of the parallel arrangement of the CSF outflow resistance and shunt. Acta Neurochir Suppl 81:23-26, 2002

19. Traboulsi R, Avan P: Transmission of infrasonic pressure waves from cerebrospinal to intralabyrinthine fluids through the human cochlear aqueduct: Non-invasive measurements with otoacoustic emissions. Hear Res 233:30-39, 2007

20. van Veelen-Vincent ML, Delwel EJ, Teeuw R, Kurt E, de
Jong DA, Brocaar MP, et al: Analysis of hearing loss after shunt placement in patients with normal-pressure hydrocephalus. J Neurosurg 95:432-434, 2001

21. Weerakkody RA, Czosnyka M, Schuhmann MU, Schmidt E, Keong N, Santarius T, et al: Clinical assessment of cerebrospinal fluid dynamics in hydrocephalus. Guide to interpretation based on observational study. Acta Neurol Scand 124:85-98, 2011

22. White KR, Vohr BR, Maxon AB, Behrens TR, McPherson MG, Mauk GW: Screening all newborns for hearing loss using transient evoked otoacoustic emissions. Int J Pediatr Otorhinolaryngol 29:203-217, 1994

23. Winston KR, Lopez JA, Freeman J: CSF shunt failure with stable normal ventricular size. Pediatr Neurosurg 42:151155,2006

\section{Disclosure}

The authors report no conflict of interest concerning the materials or methods used in this study or the findings specified in this paper.

\section{Author Contributions}

Conception and design: Avan, Chazal. Acquisition of data: Avan, Sakka, Chomicki, Gabrillargues, Khalil. Analysis and interpretation of data: all authors. Drafting the article: Avan, Sakka, Chazal. Critically revising the article: Avan, Sakka, Gabrillargues. Reviewed submitted version of manuscript: Avan, Sakka. Approved the final version of the manuscript on behalf of all authors: Avan. Statistical analysis: Avan, Chomicki. Administrative/technical/material support: Avan, Gabrillargues, Khalil, Chazal. Study supervision: Avan, Sakka, Chazal.

\section{Correspondence}

Paul Avan, Neurosensory Biophysics (UMR INSERM 1107), School of Medicine, 28, Place Henri Dunant, Clermont-Ferrand 63000, France.email: paul.avan@udamail.fr. 